\title{
Soft Tissue Morphometry of the Malleus-Incus Complex from Micro-CT Imaging
}

\author{
Jae Hoon Sim ${ }^{1,2}$ and Sunil Puria ${ }^{1,2,3}$ \\ ${ }^{1}$ Department of Mechanical Engineering, Stanford University, Stanford, CA 94305, USA \\ ${ }^{2}$ Department of Veterans Affairs, Palo Alto, CA 94304, USA \\ ${ }^{3}$ Department of Otolaryngology—Head and Neck Surgery, Stanford University, Stanford, CA 94305, USA
}

Received: 29 December 2006; Accepted: 11 October 2007

\begin{abstract}
The malleus-incus complex (MIC) is unique to mammalian hearing. To develop a comprehensive biomechanical MIC model for the human middle ear, measurements regarding its anatomical features are a necessity. Micro-scale X-ray computed tomography (micro-CT) imaging, which is known to be a suitable method for imaging high-density tissue such as middle-ear ossicles and surrounding bones, is used in this study to determine the three-dimensional (3-D) morphometry of the soft tissue attachments of the MIC. The MIC morphometry is based on their 3-D reconstruction from micro-CT image slices with resolutions ranging from 10 to $20 \mu \mathrm{m}$. The suspensory ligament and tendon attachments of the malleus and the incus as well as the incudomalleal joint (IMJ), are quantified in terms of dimensions, positions, and orientations for four human cadaver temporal bones. The malleus principal frame, the incus principal frame, and the MIC principle frame are calculated and the morphometry is reported in relation to each of these frames for the first time. The resulting values show significant variation across ear samples, suggesting that models of the MIC should be based on individual anatomy. The IMJ morphometry dimensions appear to be proportional to the ossicular mass. The micro-CT imaging modality is a nondestructive and relatively fast method for obtaining soft tissue morphometry and provides accurate anatomical features in relation to the principal axes of bones.
\end{abstract}

Correspondence to: Sunil Puria - Department of Mechanical Engineering - Stanford University - Stanford, CA 94305, USA. Telephone: +1-650-7238089; fax:+1-650-7231778; email: puria@stanford.edu
Keywords: morphometry, middle ear, soft tissue, suspensory attachment, tensor tympani tendon, ligament, malleus, incus, malleus-incus complex, incudomalleal joint (IMJ), computed tomography (CT), micro-CT

\section{INTRODUCTION}

Among vertebrates, one of the most distinguishing traits of mammals is the presence of three middle ear bones and this distinction is used as a criterion for including fossilized or living animals in the mammalian class (Masterton et al. 1969; Colbert and Morales 1991). The middle ear bones consisting of the malleus, incus, and stapes, which are the smallest bones in the human body, are attached to surrounding bony structures by suspensory attachments such as ligaments and tendons and are connected to each other by two joints. The anatomical features include the morphometry of the middle-ear bones as well as the morphometry of the soft tissues. Inertial properties of the middle-ear bones for human (Kirikae 1960; Beer et al. 1999; Weistenhöfer and Hudde 1999; Puria et al. 2007a; Sim et al. 2007) and for cat, guinea pig, and chinchilla (Puria et al. 2007a) have been reported previously. However, detailed morphometry of the soft tissues such as their dimensions and relative positions mostly remains unknown.

Several general descriptions of the suspensory attachments of the malleus have been reported (Gray 1918; Schuknecht 1974; Beer et al. 1996; Gan et al. 2002; Koike et al. 2002). Though the number of identified suspensory attachments to the malleus has 
varied from two (Koike et al. 2002) to five (Beer et al. 1996) in previous studies, four suspensory attachments consisting of the tensor tympani tendon, the anterior attachment, the lateral attachment, and the superior attachment are generally accepted (Gray 1918; Schuknecht 1974; Beer et al. 1996; Gan et al. 2002). This is consistent with our observation of the same four attachments to the malleus under a microscope (Fig. 1) and within micro-CT images from all four ear samples used in this study.

The anterior attachment and the lateral attachment are generally described as suspensory ligaments consisting of collagen fibers, while the superior attachment is sometimes considered to be a thin fold of mucous membrane consisting of a thin layer of epithelium. Recent observations by Merchant (personal communication, 2006) indicate the possible existence of collagen fibers within the superior attachment, although they were found in only two out of the six temporal bone samples that were examined. Considering this observation, the superior attachment is referred to as the "superior attachment of the malleus" in this work, while the anterior attachment and the lateral attachment are both referred to as ligaments.

The incus ligament, which is sometimes referred to as the posterior ligament of the incus, is a short, thick band connecting the end of the short crus of the incus to the fossa incudis (Gray 1918), and has a more complicated geometry than the ligaments of the malleus. This ligament contacts the incus mainly on the medial and lateral parts of the short crus of the incus, and connects to the bony wall of the middle-ear cavity on the medial (attaching to the incus buttress) and lateral sides, so this ligament is sometimes considered to be two separate ligaments. It has been observed, however, that the two parts are connected through the inferior or the posterior parts of the short crus of the incus, so it is in fact a single continuous ligament rather than two separate ligaments. The contact areas on the middle-ear cavity wall are a little larger than the contact areas on the incus.
A

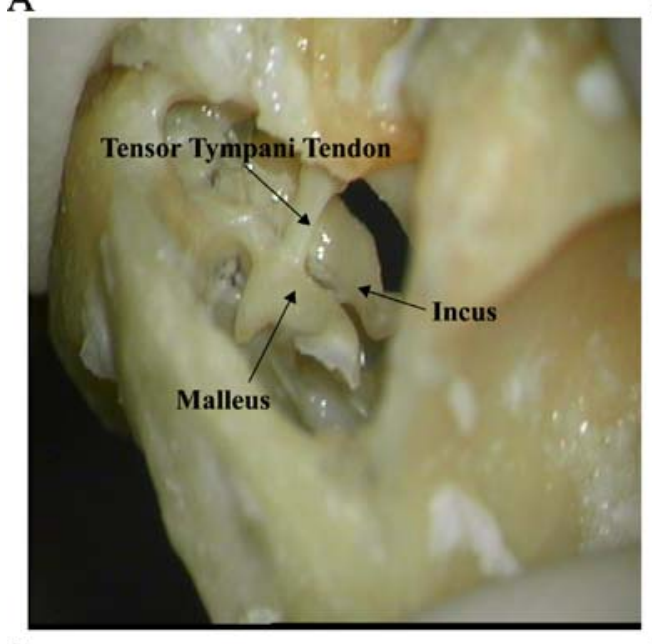

$\mathrm{C}$

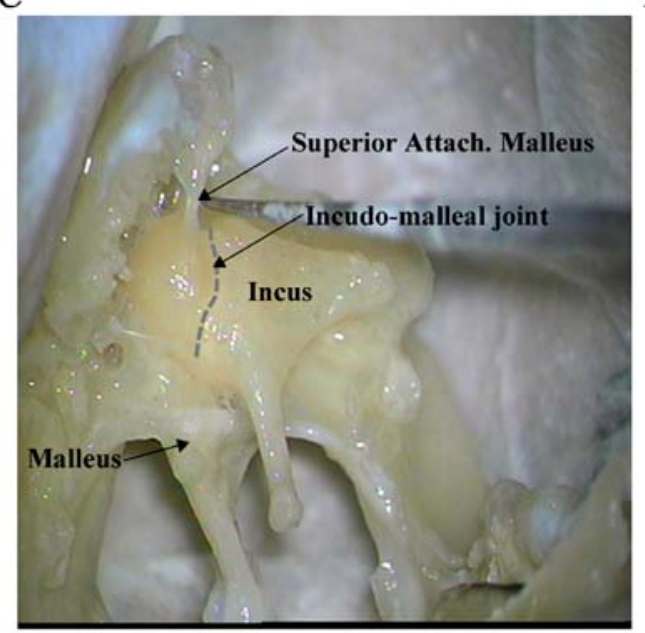

B

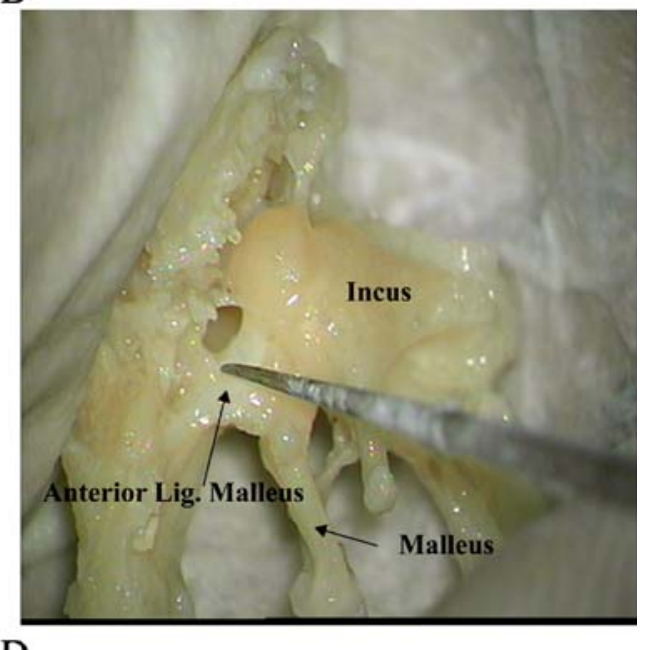

D

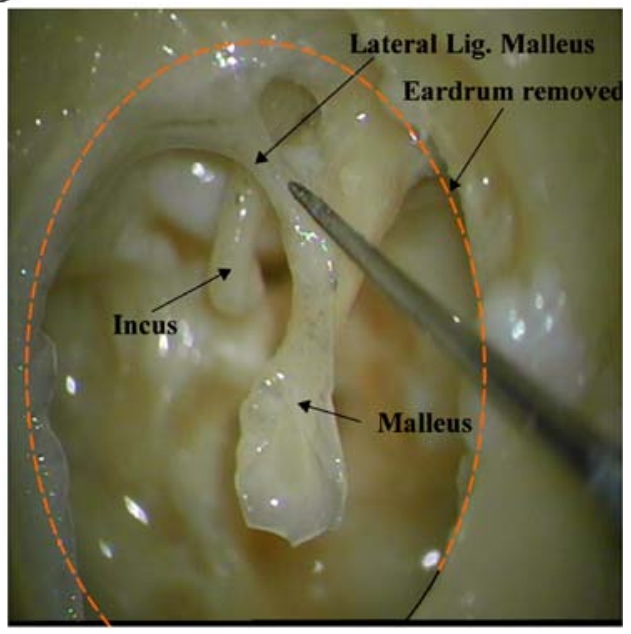

FIG. 1. Microscopic view of suspensory attachments of the malleus in an isolated malleus-incus preparation (ear 4, a right ear). A Tensor tympani tendon (anterior view), B anterior ligament (medial view), C superior attachment (medial view), and D lateral ligament (lateral view). 
The incudomalleal joint (IMJ), which has a twisted saddle shape as first described by Helmholtz (1968), has been considered to be diarthrodial joint binding the malleus and the incus at its two articular facets (Schuknecht 1974; Marquet 1981). It is also known that the two articular surfaces are connected by thin fibrous structures along the boundary. These fibrous structures are thickened on its medial and lateral aspects, where the gap between the two surfaces is relatively large. The joint is presumed to contain synovial fluid inside the capsule (Marquet 1981). All these descriptions are consistent with our observations under a microscope and of scanning electron microscopy (SEM) photos of the joint interface (Fig. 2).

Many folds of peritoneum and other membranes are also found to be attached to the MIC, which provide pathways for the blood supply to the ossicles (Schuknecht 1974). These tissues are believed to have low stiffness and thus minimal influence on the MIC motion. They are not included in this study because the focus of this work is on the soft tissues that are likely to affect the MIC dynamics.

To obtain three-dimensional anatomical information about the soft tissues in the human middle ear, traditional histological methods have been used, but they are destructive and require several months for results to be obtained. Physiological measurements of the middle ear are often made with different ossicular manipulations including ossicular disarticulation and removal of the eardrum (Sim et al. 2006). Another issue in modeling the middle ear is the fairly large variations in middle-ear anatomy across ear samples. To formulate an accurate biomechanical model, individual anatomy may be important, which requires a fast and non-destructive method. This work shows that micro-CT imaging, which is known to be a suitable method for high-density tissue such as the ossicles and surrounding bone (Decraemer et al. 2003; Lane et al. 2004; Sim et al. 2006; Puria et al. 2007a), can also be used to image the soft tissues of the malleus-incus complex (Figs. 3 and 4).

This work is one step toward characterizing the biomechanics of the malleus-incus complex. To decouple the MIC from eardrum mechanics and the stapes mechanics, we have developed an isolated MIC preparation where the eardrum and the stapes are removed (Fig. 3 and Fig. 4 right panel). The resulting morphometry data are important for anatomically based 3-D computational models of the MIC subsystem of the human middle ear (Sim et al. 2006).
A

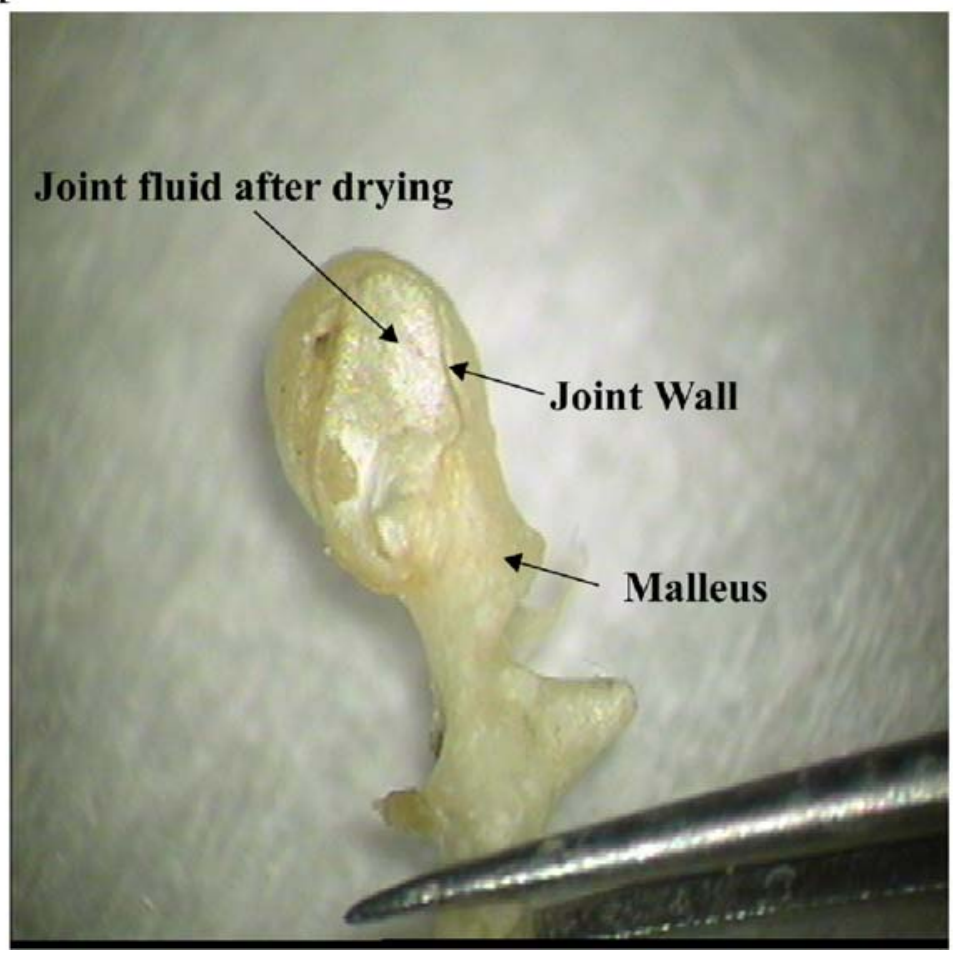

B

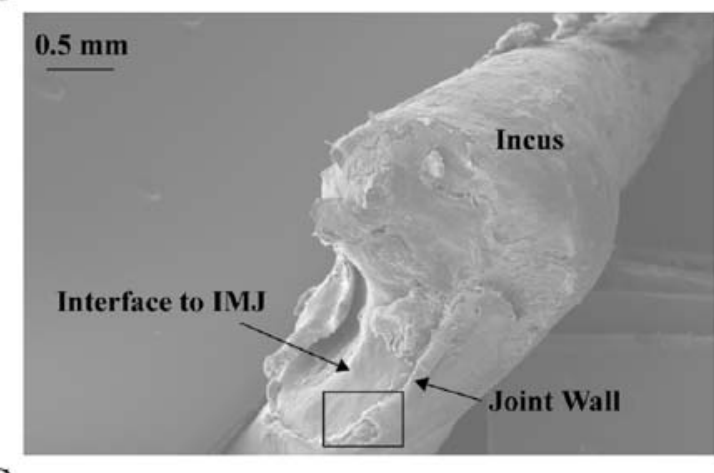

C

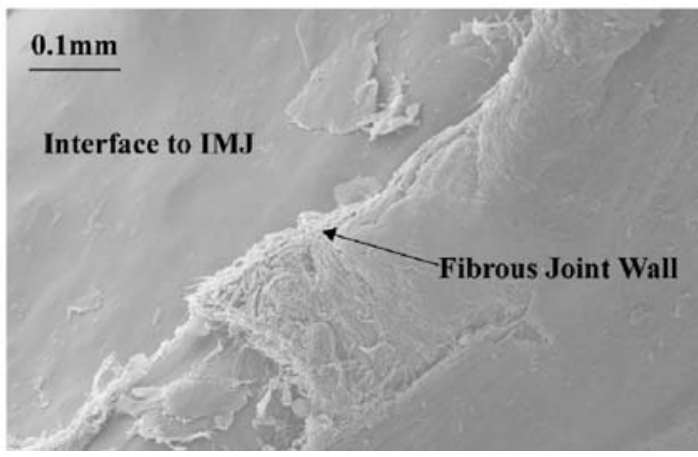

FIG. 2. The incudomalleal joint (IMJ) interface on the malleus and incus. A Microscopic view after drying of the joint fluid for $30 \mathrm{~min}$, B SEM photo of the interface to the incus, and C SEM photo of the fibrous structure at the joint wall. The joint is filled with sticky fluid, which is contained by the fibrous wall along its boundary. 


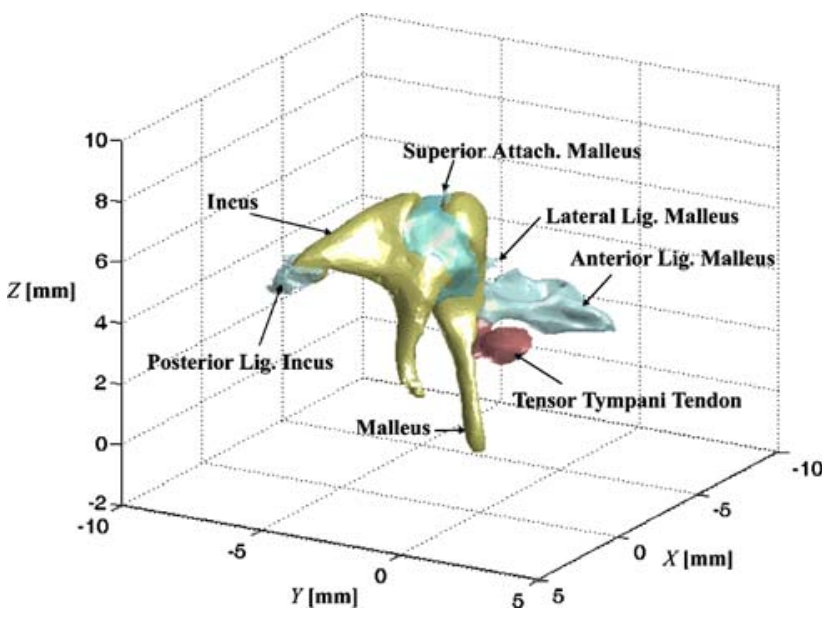

FIG. 3. Isolated malleus-incus complex (MIC) of the human middle ear obtained from 3-D reconstruction of micro-CT scan images. Blue structures indicate suspensory attachments while the tensor tympani tendon is shown in red. The middle-ear cavity walls, where the attachments terminate, are not shown. The umbo is at the origin of the $X, Y$, and $Z$ axes of the $\mathrm{CT}$ frame.

\section{MATERIALS AND METHODS}

\section{Temporal bone preparation}

Four human temporal bones from four different human cadavers were used (three right ears and one left ear). The temporal bones were harvested within 2 days after death and were frozen immediately upon extraction. The ear canal and the middle-ear cavity were opened widely to minimize X-ray attenuation and to reduce the specimen size so as to allow the scan resolution to be increased. Because this study was originally planned to obtain anatomical information for a model of the isolated MIC dynamics, the eardrum and stapes were dissected using a surgical laser (AccuSTAT 10-6110).

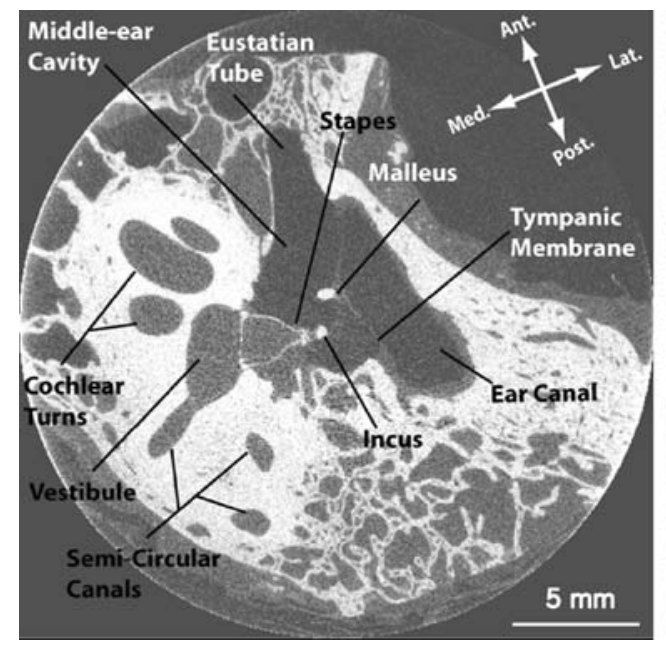

FIG. 4. Micro-CT images of an intact ear (left) and the isolated MIC (right). These images were obtained from $12.5 \mu \mathrm{m}$ iso-volume scans with the $25.6 \mathrm{~mm}$ diameter holder (intact ear, left) and $10.5 \mu \mathrm{m}$ iso-

\section{Micro-CT imaging}

The vivaCT 40 micro-CT scanner developed by SCANCO Medical AG (www.scanco.ch) was used for this study. Figure 4 shows slice images of an intact ear (left) and the isolated malleus-incus complex (right). The best resolution of $10.5 \mu \mathrm{m}$ could be obtained by reducing our specimen size to fit into the 21.5-mm diameter holder (Sim et al. 2007).

The maximum X-ray intensity setting of $145 \mu \mathrm{A}$ was used to get sufficiently good signal-to-noise ratio and good image clarity (Sim et al. 2007). By increasing the integration time up to $2,000 \mathrm{~ms}$ which corresponds to measurement times of 9-12 h for most of our preparations, it was possible to further improve soft tissue visibility.

The vivaCT 40 micro-CT scanner in this study allows 45,55 , or $70 \mathrm{keV}$ as the diagnostic energy level. We had difficulty in differentiating soft tissue from the surrounding background air in grayscale with $70 \mathrm{keV}$ because of small attenuation difference between them. With the photon energy level set to $45 \mathrm{keV}$, the attenuation difference between bone and air was larger, but visibility of soft tissue, which is the most difficult part, was not improved, and even partially degraded compared to the scan with the photon energy level set to $55 \mathrm{keV}$ (Fig. 5).

We also observed some interruptions because of "beam hardening" effects (Brook et al. 1976; Wang et al. 1998) in large bony sections with the photon energy level set to $45 \mathrm{keV}$. This led us to select $55 \mathrm{keV}$ as the photon energy, where soft tissue was clearly distinguishable from its background and grayscale differences between bone and the surrounding air and soft tissue are sufficiently large.

Another factor affecting the visibility of soft tissue is the imaged area of the soft tissue in each slice,

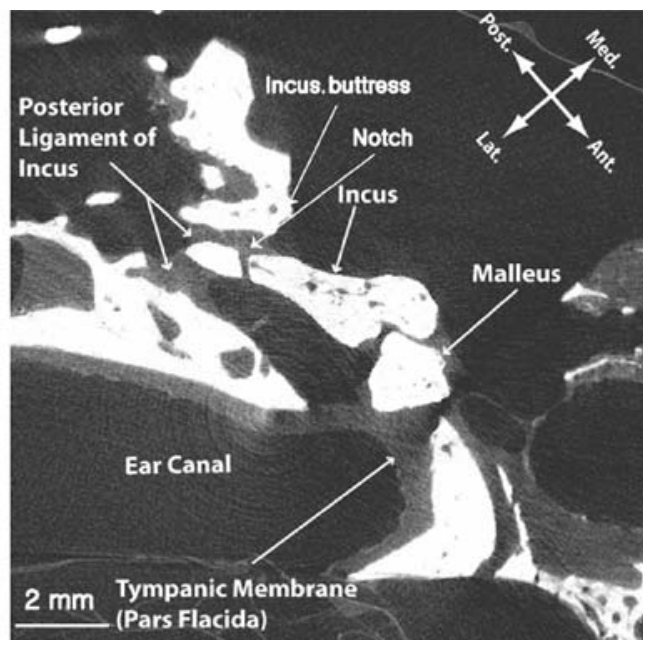

volume scans with the $21.5 \mathrm{~mm}$ diameter holder (the isolated MIC, right). Note the notch in the posterior part of the incus. (Reprinted with permission from Sim et al. 2007). 


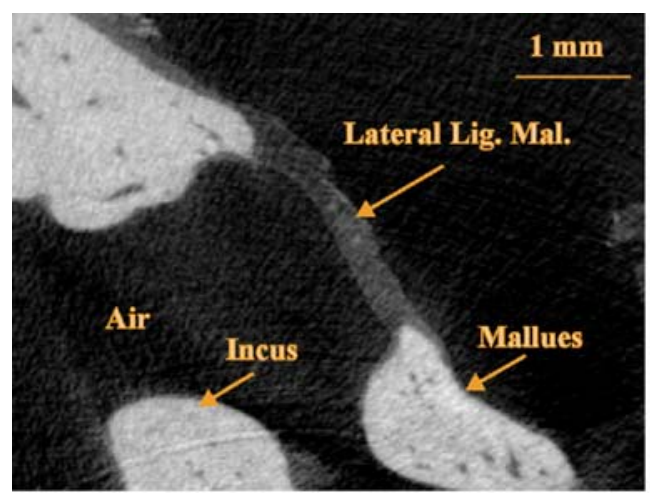

FIG. 5. Micro-CT images with a photon energy level of $55 \mathrm{keV}$ (left) and $45 \mathrm{keV}$ (right). With a photon energy level of $45 \mathrm{keV}$, attenuation differences between bony portion and its background

where smaller areas yield lower visibility. To avoid the small areas associated with suspensory tissues intersecting the image planes longitudinally, and to ensure that all suspensory tissues are clearly visible, scans for each specimen are made in two approximately orthogonal directions: superior-inferior and anterior-posterior directions.

\section{Segmentation and three-dimensional volume reconstruction}

The Evaluation Program (v.6.0) provided by the microCT manufacturer (SCANCO, Switzerland) was used for segmentation and 3-D volume reconstruction from the stacks of slice images. The first step in using this program is to outline the object of interest with contours in each slice image. For bone which has a high contrast ratio with respect to surrounding soft tissue and air, contouring can be performed by automated software algorithms as follows: a contour (which approximately matches the shape of the bone) is initially drawn on a slice by hand, and then a "shrink-wrapping" algorithm based on Gaussian segmentation is used to automatically adjust the shape to closely match the edge of the bone. The resulting contour is then copied to the next slice (iterating forwards) or the previous slice (iterating backwards), and the shrink-wrapping algorithm is repeated. Because the grayscale differences between soft tissue and air are too small (Fig. 6A), automatic contouring cannot be used for soft tissue, and hand segmentation is required. Once the object is separated from adjacent objects by contours, a range in grayscale is applied to identify "full" voxels and "empty" voxels, which correspond to the volume within the range and outside of the range. Different grayscale ranges are selected for objects of high and low grayscale values corresponding to bone and soft tissue (Fig. 6B), which are readjusted for each scan. These ranges are not typically the same for different specimens and scans

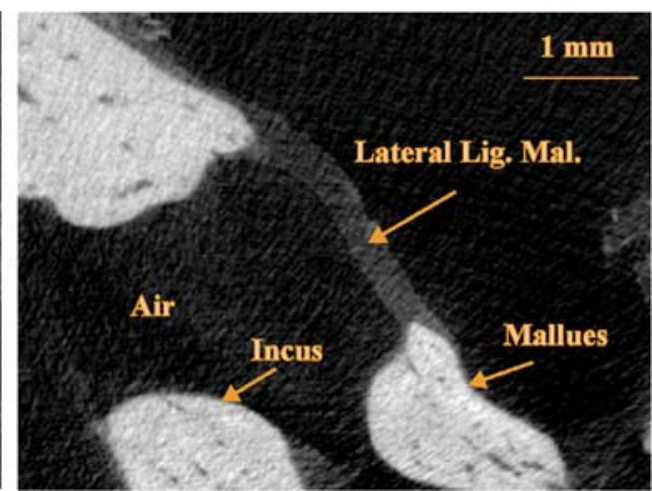

were larger, but the visibility of soft tissue was not improved, and even partially degraded compared to the scan with the a photon energy level of $55 \mathrm{keV}$.

because even the same material can have a different grayscale value depending on the scan conditions and specimen preparation.

Figure $6 \mathrm{~B}$ shows the grayscale values along the red line in Figure $6 \mathrm{~A}$, which were recalculated such that the maximum attenuation $\left(\mu / p=8 \mathrm{~cm}^{2} / \mathrm{g}\right)$ corresponds to 1,000 and no attenuation $(\mu / p=0)$ corresponds to 0 . Based on a histogram analysis (Fig. 6C), a grayscale range of 200-350 was used for the soft tissue with our scan condition and specimen preparation. A range above 350 was used for bone and a range below 200 was used for surrounding air. After segmentations are done for a stack of slices, segmented slices are combined to reconstruct the 3-D volume of the object.

\section{Registration into the principal frame}

To describe attachment positions and orientations of suspensory attachments, all reconstructed 3-D volumes are registered into the principal frames of the malleus, the incus, or the combined malleus-incus complex. Each principal frame consists of the corresponding principal axes and has the corresponding center of mass as its origin.

Portions of the malleus/incus bones are vascularized and thus contain lower-density blood vessels (Donaldson et al. 1992). Consequently, the entire bone cannot be treated with uniform density (Fig. 7).

The center of mass and inertia in the Cartesian coordinate system are calculated by the standard discretization of 3-D volume information from the micro-CT images. The specific techniques and results for the same ears reported in this study are described elsewhere (Puria et al. 2007a; Sim et al. 2007). The bone mass, which includes the mass of the lowdensity blood vessels as well as the mass of the highdensity bony parts, is physically measured using a scale (ACCULAB LA-110) with a precision of $0.1 \mathrm{mg}$ (Table 1). All mass measurements are made $15 \mathrm{~min}$ after the bone is removed from a saline solution bath. 
A

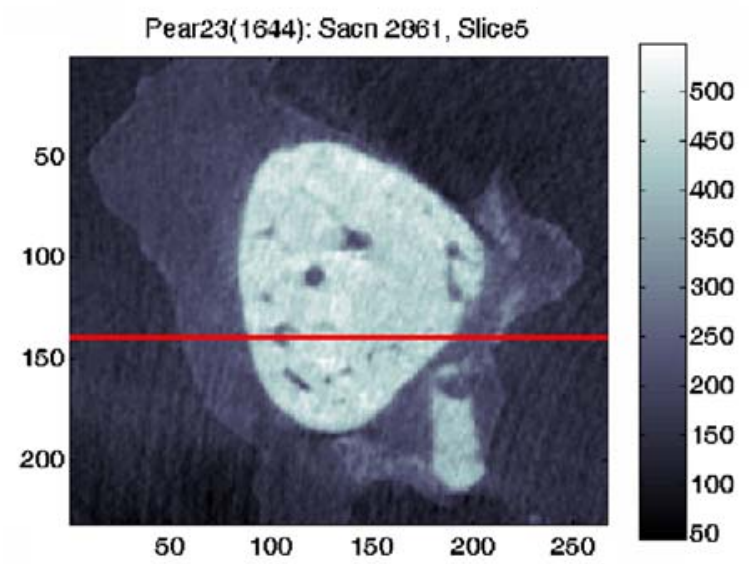

B

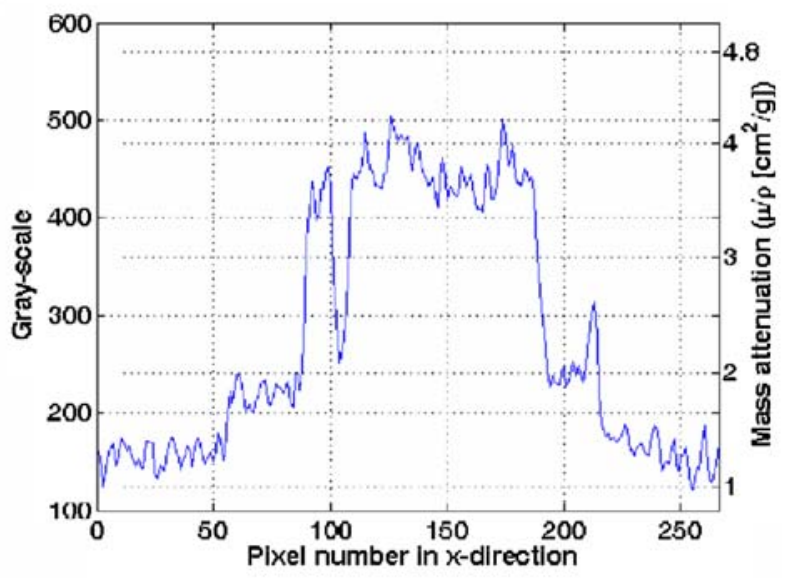

$\mathrm{C}$

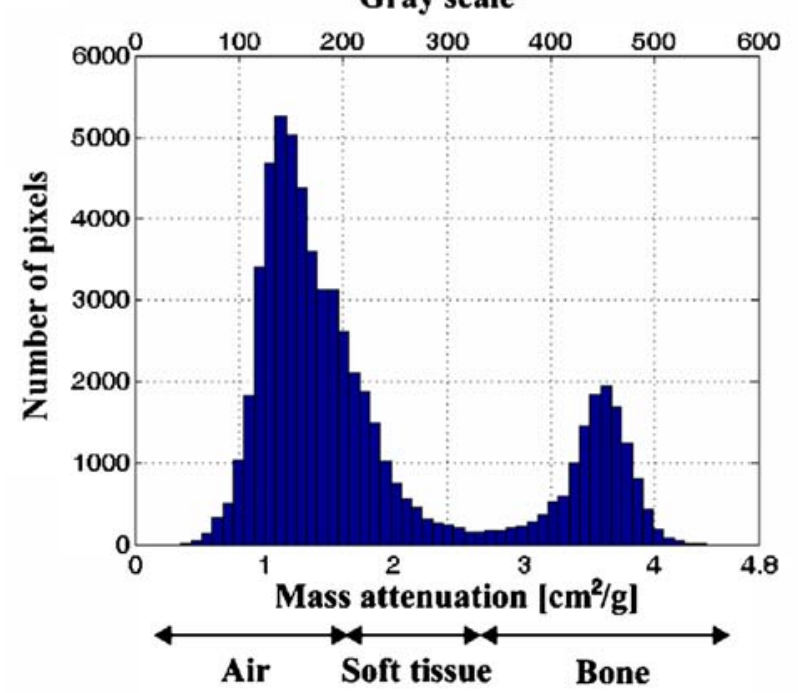

FIG. 6. Grayscale levels of a micro-CT image. A Slice image of the malleus, surrounding tissue, and air, $\mathbf{B}$ grayscale values along the red line on $\mathbf{A}$, and $\mathbf{C}$ histogram of the scan image $\mathbf{A}$. Bone and soft tissue are distinguishable from surrounding air (A). The grayscale values for bone have a higher rang (350-550) than soft tissue (200-350) and air (below 200). (Reprinted with permission from Sim et al. 2007).
The density of blood vessels is assumed to be the same as the density of water $\left(1,000 \mathrm{~kg} / \mathrm{m}^{3}\right)$.

Once moments of inertia are known for a given frame, the orientation of the principal frame is calculated such that all nondiagonal terms in the inertia matrix are zero simultaneously (Sim et al. 2007). The three orthogonal principal axes approximately lie along the anterior-posterior direction, the inferior-superior direction, and the lateral-medial direction. The principal axis in the anterior-to-posterior direction is set as $X$-axis, the principal axis in the inferior-to-superior direction as $Y$-axis, and the principal axis in the lateral-to-medial direction as Z-axis. This designation of axes results in a "right-hand-rule" Cartesian coordinate system for right ears and a "left-hand-rule" Cartesian coordinate system for left ears, making comparison between ear samples possible regardless of whether the ear sample is from a left or a right ear. Figure 8 shows the malleus and its suspensory attachments aligned to the malleus principal frame (A) and the malleus-incus complex and its suspensory attachments aligned to the MIC principal frame (B).

\section{Parametric characterization of soft tissue morphometry}

Because of poor signal-to-noise surrounding the soft tissue and hand segmentation errors, the 3-D volume reconstruction of the MIC suspensory attachments, shown in Figure 9 in light blue, are too rough to be incorporated into our relatively simple beam-like descriptions of the suspensory attachments. In a vibrating system, each suspensory attachment can be modeled as three translational and three rotational springs when its attachment to the vibrating body is distributed over a relatively small area compared to the size of the body. If the length of the suspensory attachment is relatively large compared to its crosssectional dimension, it can be approximated by a beam structure such as a tapered cylinder. The 3-D volumes of suspensory attachments of the malleus are described to a first-order approximation as tapered cylinders with equivalent diameters, which are calculated from the actual cross-sectional areas of the suspensory attachments and are shown in Figure 9 in red.

In determining the equivalent diameters of the suspensory attachments for the mathematical model, the volume fraction and alignment of fibers within the suspensory attachment should also be considered. In this study, the focus is on the geometry of suspensory attachments, however, so the equivalent diameters are calculated such that the tapered cylinders have approximately the same cross-sectional area as the actual corresponding suspensory attachment.

To describe the attachment positions and orientations, simplified 3-D volumes of the suspensory 

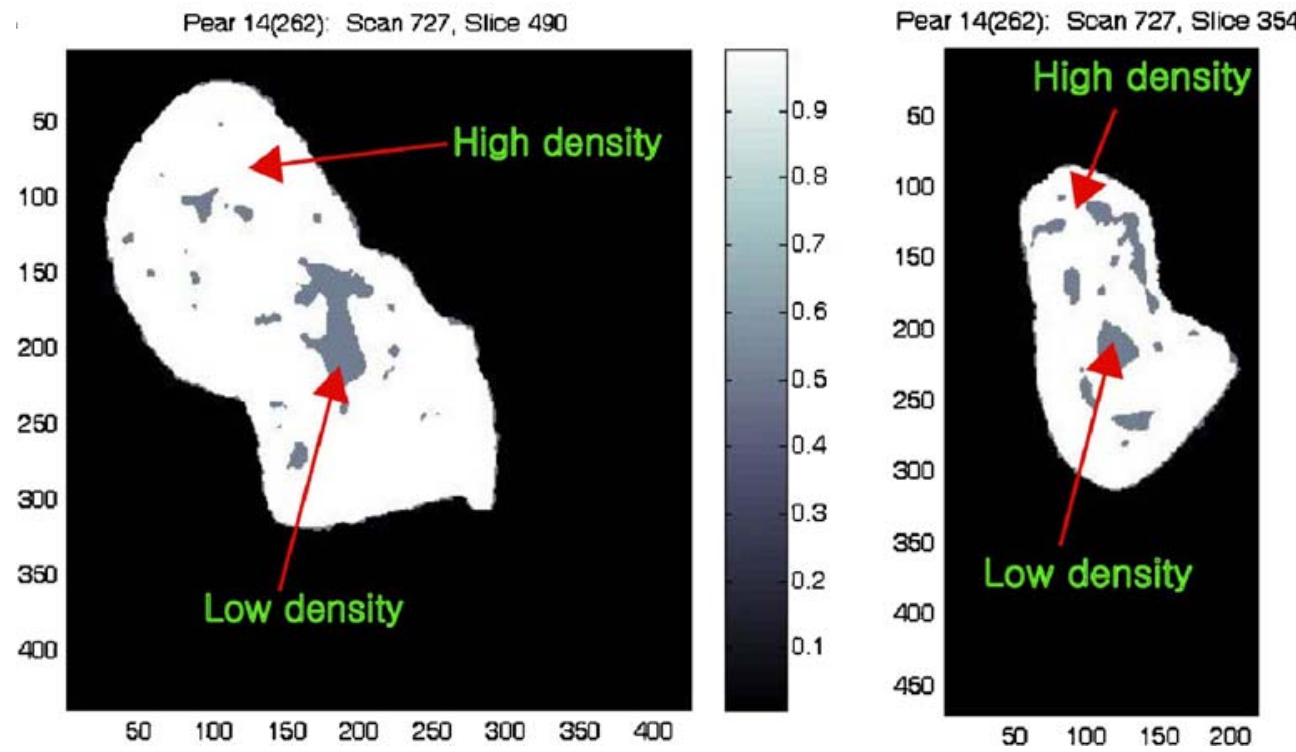

FIG. 7. Segmented slice image of malleus (left) and incus (right). These bones contain "high-density" bony parts and "low density" parts consisting of blood vessels. For inertial calculation, the density

attachments of the malleus are registered into the malleus principal frame, and into the MIC principal frame (Fig. 10).

The attachments to the malleus are described by position vectors from the corresponding center of mass to the attached location, and their orientations are described by unit-directional vectors which start at the malleus attachments and go to the bony middle-ear cavity attachments.

To characterize the incus ligament morphometry, average thicknesses and contact areas on the medial and lateral sides of the incus are measured from the 3-D solid model. The orientation of the incus ligament is described in the incus or MIC principal frames by the orientations of two vectors normal to the planes contacting the lateral and medial sides of the incus. Figure 11 shows the two contact areas along with the planes that are calculated as the best fits to the areas, in addition to their corresponding normal vectors. The planes are calculated using the commercial program RapidForm (www.rapidform.com), and of the blood vessel is assumed to be the density of water, and the bone mass is measured using a precision scale. (Reprinted with permission from Sim et al. 2007).

the attachment positions on the incus are described by the centroid positions of the two contact areas in the principal frames.

The incudomalleal joint (IMJ) is characterized by its maximum and minimum thicknesses, width, area in contacting the malleus and incus, and total volume. To determine the thickness map of the IMJ, the distance transformation (DT) method (Hildebrand and Rüegsegger 1997), which measures the mean thickness of a structure by filling it with spheres of the largest possible diameter and calculating their mean diameter, is used.

\section{RESULTS}

Dimensions, positions, and orientations of suspensory attachments for the malleus principal frame are summarized in Figure 12A and for the malleus-incus principal frame in Figure 12B. The umbo position and the center of mass (at the origin) are also shown

\section{TABLE 1}

Mass and average density of the malleus, incus, and the combined malleus and incus

\begin{tabular}{|c|c|c|c|c|c|c|c|}
\hline & & Ear 1 & Ear 2 & Ear 3 & Ear 4 & Mean & SEM \\
\hline \multirow[t]{2}{*}{ Malleus } & Mass (mg) & 25.9 & 29.8 & 35.1 & 26.2 & 29.3 & 2.14 \\
\hline & $\operatorname{Density}^{\mathrm{a}}\left(\mathrm{mg} / \mathrm{mm}^{3}\right)$ & 2.14 & 2.68 & 2.35 & 2.09 & 2.31 & 0.134 \\
\hline \multirow[t]{2}{*}{ Incus } & Mass (mg) & 29.4 & 27.8 & 38.7 & 25.0 & 30.2 & 2.97 \\
\hline & Density $^{\mathrm{a}}\left(\mathrm{mg} / \mathrm{mm}^{3}\right)$ & 2.02 & 2.23 & 2.21 & 2.11 & 2.14 & 0.049 \\
\hline \multirow[t]{2}{*}{ MIC } & Mass (mg) & 55.3 & 57.6 & 73.8 & 51.2 & 59.5 & 4.96 \\
\hline & Density $^{\mathrm{a}}\left(\mathrm{mg} / \mathrm{mm}^{3}\right)$ & 2.07 & 2.44 & 2.27 & 2.10 & 2.22 & 0.086 \\
\hline
\end{tabular}

All mass measurements were made 15 min after the bone was removed from a saline solution bath.

${ }^{a}$ Average density assuming the lower density of the blood vessel is equal to the density of water. 
A

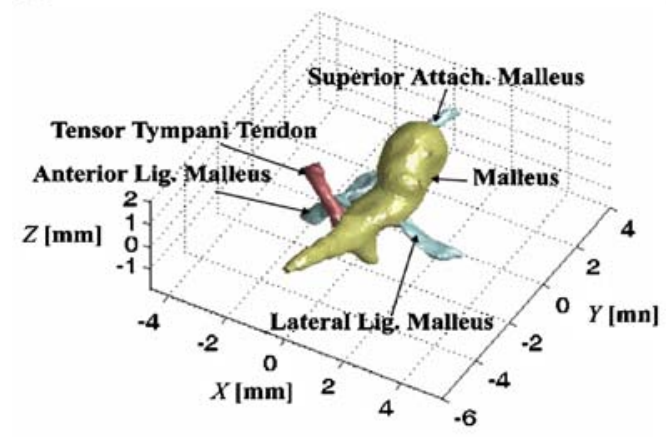

FIG. 8. Registration into the principal frame (ear 4). The principal axis in the anterior-to-posterior direction is set as $X$-axis, the principal axis in the inferior-to-superior direction as $Y$-axis, and the principal axis in the lateral-to-medial direction as $Z$-axis. The origin

in the figure. Detailed morphometry analyses, which includes mean and standard error of mean (SEM) values, are reported primarily in tabular form and are described in the sections that follow.

\section{Suspensory attachments of the malleus}

Table 2 shows the diameter and length ranges of the simplified suspensory attachments of the malleus.

\section{A}

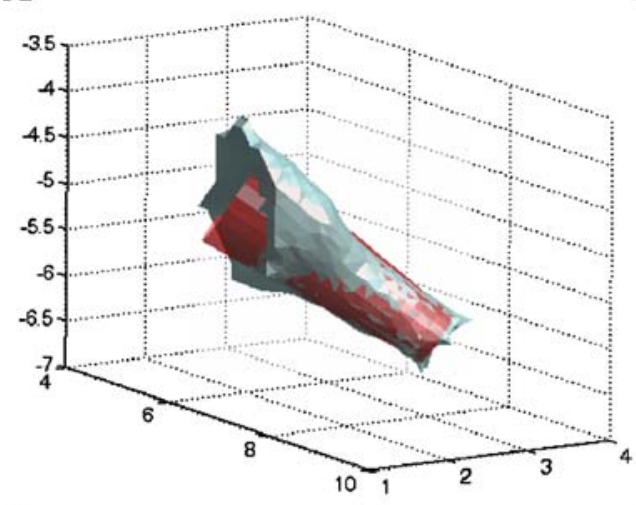

$\mathrm{C}$

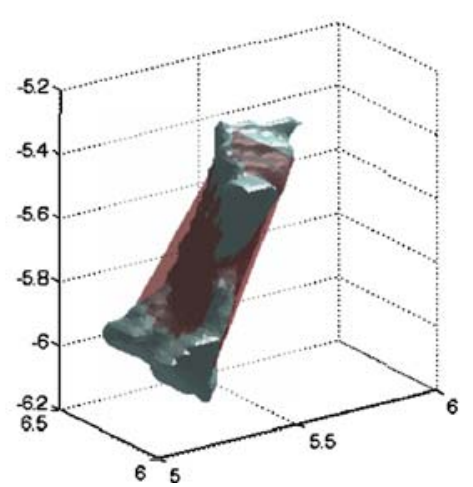

FIG. 9. Volume reconstruction of suspensory attachments of the malleus and corresponding tapered cylindrical approximation (ear 3). A The tensor tympani tendon, $\mathbf{B}$ the anterior ligament, $\mathbf{C}$ the

B

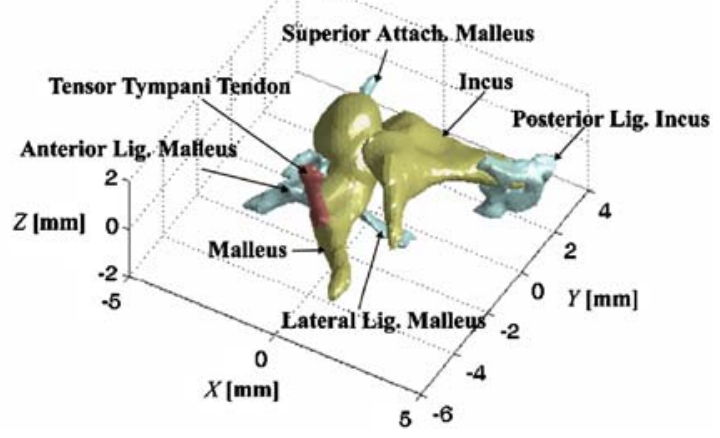

of each principal frame is the corresponding center of mass. A The malleus and its suspensory attachments aligned on the malleus principal frame and $\mathbf{B}$ the malleus-incus complex and its suspensory attachments aligned on the MIC principal frame.

The diameter across the tensor tympani tendon is nearly constant for ears 3 and 4, but it varies for ears 1 and 2. The mean minimum diameter is $0.7 \mathrm{~mm}$ at the manubrium attachment, while the mean diameter on the middle-ear cavity bone attachment is 0.9 $\mathrm{mm}$. The mean length of the tensor tympani tendon is $2.2 \mathrm{~mm}$, and ear 2 has the longest tensor tympani tendon $(2.6 \mathrm{~mm})$ among the four specimens. The tensor tympani tendon is attached to its muscle. In

B

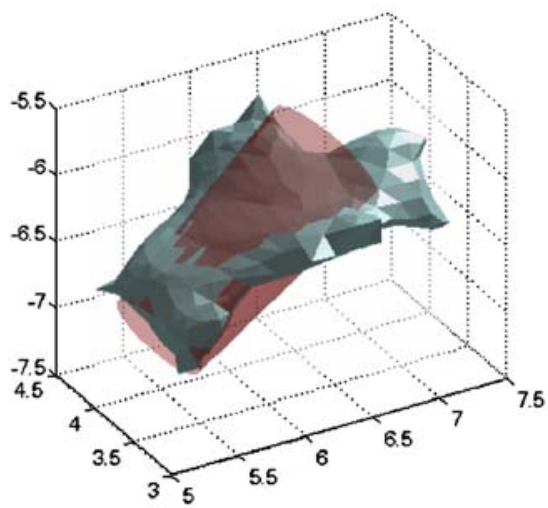

D

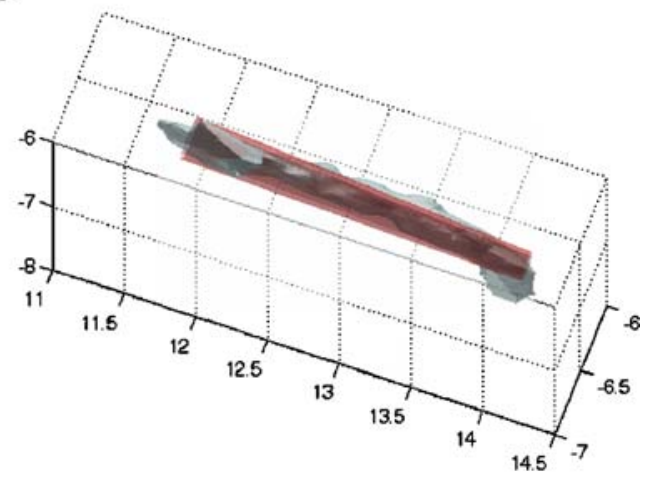

lateral attachment, and D the superior attachment. All axes dimensions are in millimeter. 


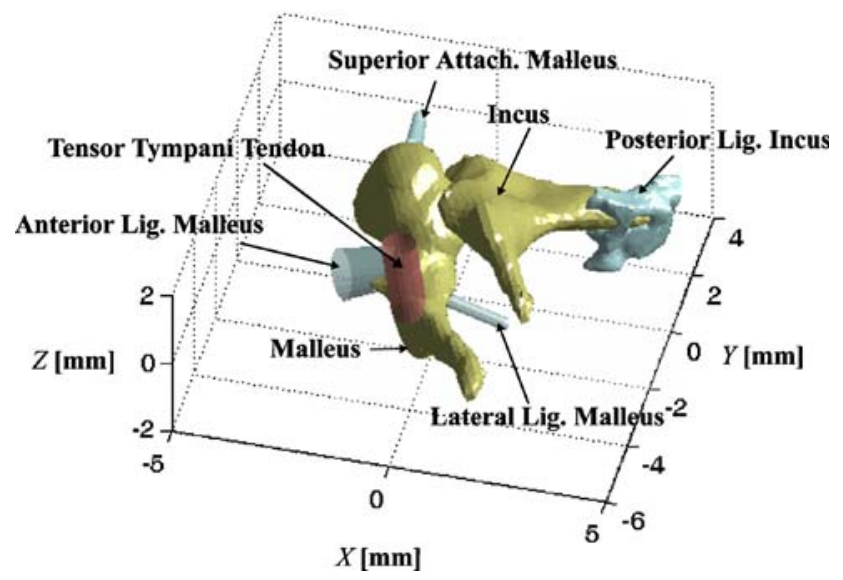

FIG. 10. Simplified features of the suspensory attachments in the MIC principal frame (ear 4). The attachment positions on the malleus are described as the position vectors of the attachment locations, and their orientations are described by the unit-directional vectors extending from the malleus to the middle ear cavity walls.

A

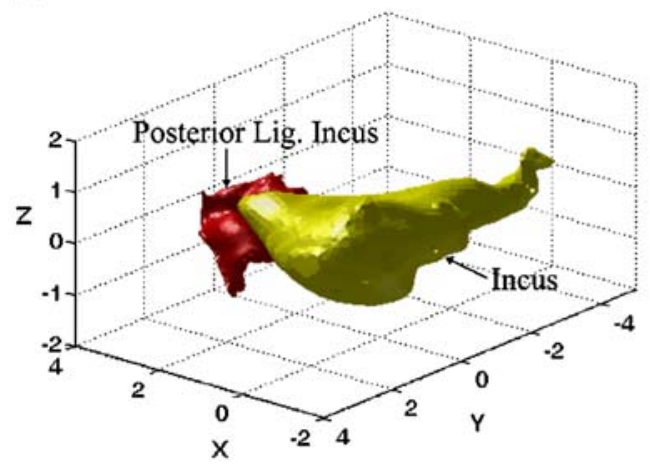

C

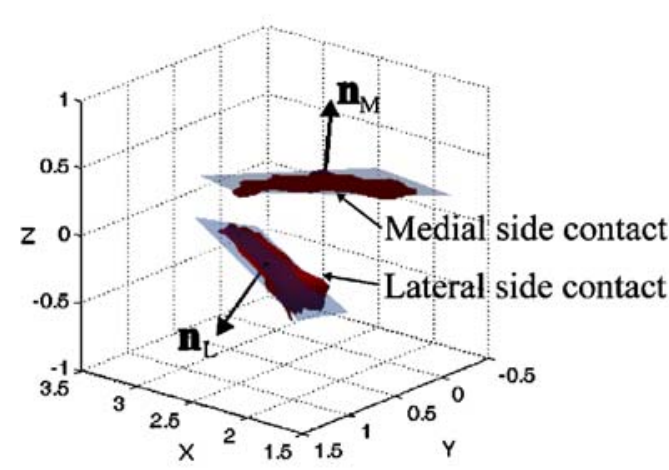

FIG. 11. Characterization of the incus ligament in the incus principal frame (ear 2). A The incus ligament aligned with respect to the incus principal frame $(X$ : anterior-to-posterior, $Y$ : inferior-tosuperior, Z: lateral-to-medial), B two contact areas of the incus ligament on the medial and lateral sides of the incus and their corresponding normal vectors, $\mathbf{C}$ planes fit to the contact areas and their two normal vectors, and $\mathbf{D}$ calculation of the planes and the normal vectors. Thickness, area, and width on the medial and lateral
Table 2, the portion of the tensor tympani tendon outside of the bone is measured as its length, which is presumed to be its tendon length. The remaining suspensory attachments of the malleus terminate on the surface of the middle-ear cavity.

The anterior ligament, which partially covers the anterior process of the malleus, has a more complicated cross-sectional shape. It has the largest cross-sectional area among suspensory attachments of the malleus, and its mean equivalent diameter is $0.8-1.0 \mathrm{~mm}$, where the equivalent diameter is calculated from the equivalent circular cross-section of the same area. The anterior ligament has a uniform equivalent diameter for ear 1 , while the diameters for ears 2, 3, and 4 vary. Ear 1, whose tensor tympani tendon has a length near the mean value, has the longest anterior ligament among the four samples, while ear 2, which has the longest tensor tympani tendon, has a short anterior ligament. Ear 4, with a short tensor tympani tendon, also has the shortest anterior ligament $(1.4 \mathrm{~mm})$, while ear 3 , which also has a
B

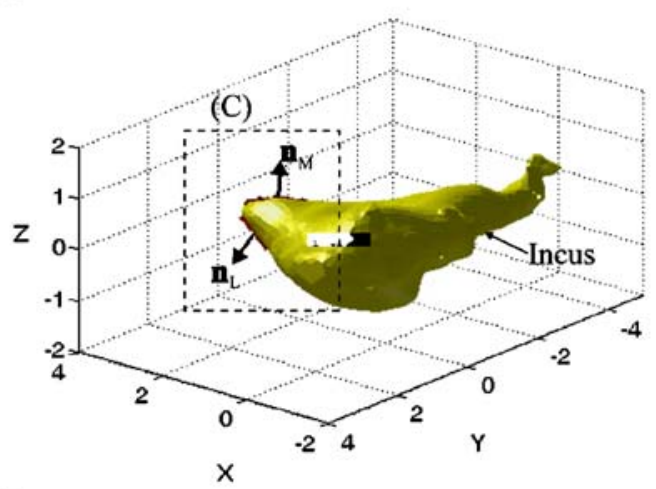

D

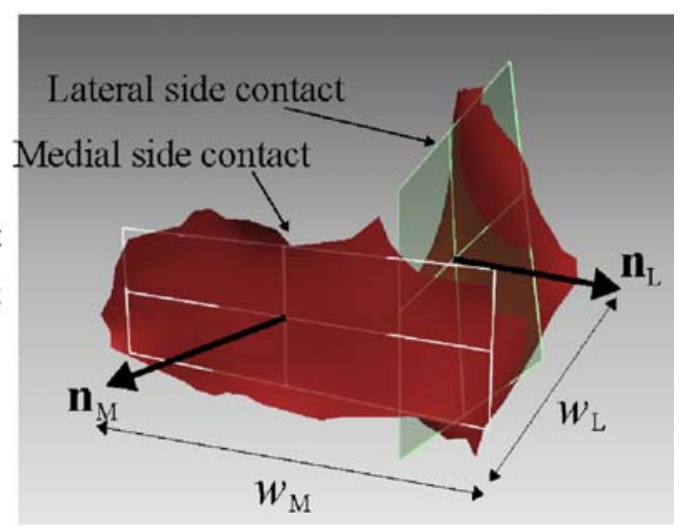

contacts are measured to quantify the dimensions of the incus ligament. Orientation of the incus ligament was described by the orientations of the two normal vectors $\left(\mathbf{n}_{M}\right.$ and $\mathbf{n}_{\mathrm{L}}$ ) in the incus principal frame and the MIC principal frame. The planes were calculated for the best fit to the contact area. The attachment positions on the incus are described by the centroid positions of the two contacting areas in the incus and the MIC principal frames. 
A

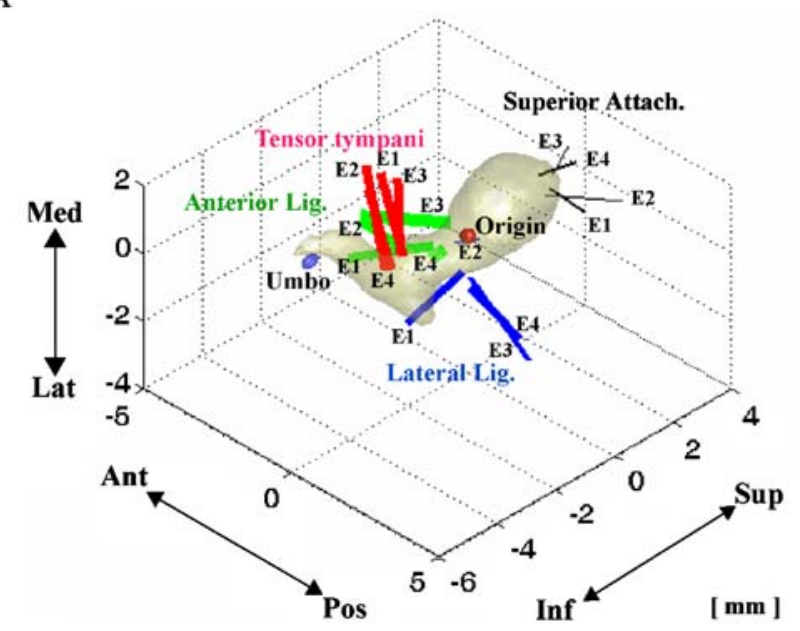

B

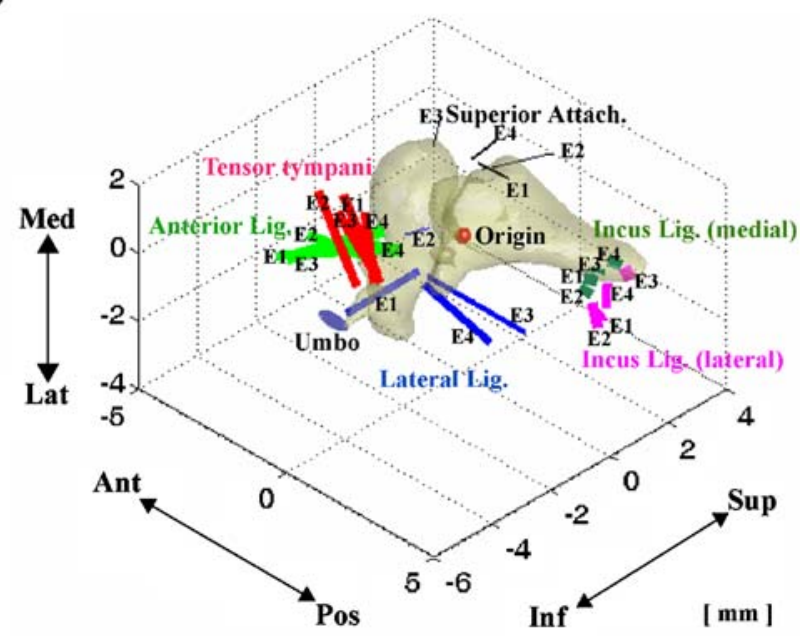

FIG. 12. Summary of length, position, and orientation of suspensory attachments in two different principal frames for the four ears measured (E1-E4). A The malleus suspensory attachments in the malleus principal frame and $\mathbf{B}$ the MIC suspensory attachments in the MIC principal frame. The bones are from ear 3. All dimensions are in millimeter. The line width is approximately proportional to the diameter of the corresponding suspensory attachment. The indicated origin is the center of mass.

short tensor tympani tendon, has a relatively long anterior ligament $(2.4 \mathrm{~mm})$. While the length of the tensor tympani tendon varies by $38 \%$ across the four ear samples, the length of the anterior ligament varies by almost $200 \%$.

The lateral ligament of ear 2 has the shape of a cylindrical bar, while for the other samples the lateral ligament is shaped either more like a rectangular plate or like a shape in-between a cylinder and a rectangular plate. For ear 2, the equivalent lateral ligament diameter $(0.2-0.3 \mathrm{~mm})$ was nearly half of the mean diameter $(0.5-0.6 \mathrm{~mm})$ and also has a relatively small length $(0.8 \mathrm{~mm})$ compared to its mean length $(2.0 \mathrm{~mm})$. The mean equivalent diameter and length of the superior attachments are small compared to other suspensory attachments of the malleus for all four ear specimens. The length is distinctly larger for ear 2 with a value more than twice that of the other specimens.

Table 3 shows the position vectors of the attachment locations of the suspensory attachments on the malleus surface in the malleus principal frame (top half of the table) and the MIC principal frame (bottom half of the table), where the origin is the corresponding center of mass in each case. In both frames, the tensor tympani tendon and the superior attachment are furthest from the center of gravity (origin), while anterior and lateral ligaments are closest. For the lateral ligament of ear 2, which has relatively small dimensions, the attachment location differs significantly from the other specimens. For the anterior ligament, variation with respect to the MIC principal frame is smaller than variation with respect to the malleus principal frame, while for the superior attachment the variation is smaller in the malleus principal frame. The attachment locations of the lateral $(1.2 \mathrm{~mm})$ and anterior $(1.0 \mathrm{~mm})$ ligaments are relatively close to the center of mass of the malleus, while the attachment locations of the tensor tympani tendon $(2.2 \mathrm{~mm})$ and the superior attachment $(2.4 \mathrm{~mm})$ are relatively far from the center of mass of the malleus. The attachment locations of the tensor tympani tendon and the anterior and lateral ligaments are further from the center of mass of the MIC than from the center of mass of the malleus, while the attachment location of the superior attachment is closer to the center of mass of the MIC $(2.3 \mathrm{~mm})$ than to the center of mass of the malleus $(2.4 \mathrm{~mm})$.

Table 4 shows the unit-directional vectors of the malleus suspensory attachments with respect to the malleus principal frame and with respect to the MIC principal frame. The tensor tympani tendon orientation shows a small variation (with an average angle deviation of $7.6^{\circ}$ from the mean vector within the malleus principal frame), while the lateral ligament shows a relatively large variation (with an average angle deviation of $30.6^{\circ}$ from the mean vector within the malleus principal frame). It is noted that it is difficult to parameterize the lateral ligament of the malleus because its attachment to the malleus varies from the anterior part to the posterior part and its orientation is also quite different depending on the ear sample. The lateral ligament starts from the malleus attachments and extends in the lateralanterior direction for ears 1 and 2, but in the lateral-posterior direction for ears 3 and 4 . The tensor tympani tendon lies on the $X Z$ plane of the MIC principal frame, that is, perpendicular to the $Y$-axis (inferior-to-superior direction), while the superior attachment is quite perpendicular to $X Z$ 
TABLE 2

Diameter and length of suspensory attachments of the malleus measured from the malleus surface to the bony middle ear cavity wall

\begin{tabular}{|c|c|c|c|c|}
\hline Suspensory attachments & Tensor tympani tendon & Anterior ligament & Lateral ligament & Superior attachment \\
\hline \multicolumn{5}{|l|}{ Diameter (mm) } \\
\hline Ear 1 & $0.6-1.0$ & $0.7-0.7$ & $0.6-0.6$ & $0.2-0.3$ \\
\hline Ear 2 & $0.6-0.9$ & $0.7-0.9$ & $0.2-0.3$ & $0.2-0.2$ \\
\hline Ear 3 & $0.8-0.8$ & $0.9-1.2$ & $0.5-0.6$ & $0.1-0.2$ \\
\hline Ear 4 & $0.8-0.8$ & $0.9-1.1$ & $0.6-0.7$ & $0.3-0.4$ \\
\hline Mean & $0.7-0.9$ & $0.8-1.0$ & $0.5-0.6$ & $0.2-0.3$ \\
\hline SEM & $0.06-0.05$ & $0.06-0.11$ & 0.09-0.09 & $0.04-0.05$ \\
\hline \multicolumn{5}{|l|}{ Length (mm) } \\
\hline Ear 1 & 2.1 & 3.0 & 2.5 & 1.0 \\
\hline Ear 2 & 2.6 & 1.7 & 0.8 & 2.3 \\
\hline Ear 3 & 2.0 & 2.4 & 2.7 & 0.8 \\
\hline Ear 4 & 2.0 & 1.4 & 2.1 & 1.0 \\
\hline Mean & 2.2 & 2.1 & 2.0 & 1.3 \\
\hline SEM & 0.14 & 0.36 & 0.43 & 0.34 \\
\hline
\end{tabular}

plane of the MIC principal frame $\left(90 \pm 20.2^{\circ}\right)$. The anterior ligament lies on the $X Z$ plane of the malleus principal frame and extends from the attachment on the malleus to the anterior side. In summary, the suspensory attachments of the malleus of ears 1 and 3 have similar dimensional values, but exhibit large differences in orientation, while the suspensory attachments of ears 1 and 2 have similar orientations, but exhibit large differences in their shapes and dimensions.

\section{Incus ligament}

Table 5 shows the average thickness and contact area of the incus ligament on the medial and lateral sides of the incus. For all four specimens, the average thickness of the lateral part $(0.59 \mathrm{~mm})$ is greater than that of the medial part $(0.38 \mathrm{~mm})$. The average thickness of the lateral part is about twice as large as the average thickness of the medial part for ear 4, while the difference in thicknesses between the two for ear 3 is small $(0.09 \mathrm{~mm})$. The contact area on the lateral side of the incus is larger than the contact area on the medial side of the incus for ears 2, 3, and 4, while ear 1 has a larger contact area on the medial side of the incus. The contact area on the lateral side of the incus is about twice as large as the contact area on the medial side of the incus for ear 3, while the two contact areas for ear 4 are nearly equal. The width on the medial side contact is larger than the width on the lateral side contact for all four ear samples. The four ear samples show very small variation in the width of the contact surfaces on the medial side (the standard error of the mean, SEM, is about $1 \%$ of the mean value), while the variation is

TABLE 3

\begin{tabular}{|c|c|c|c|c|}
\hline Suspensory Attachments & Tensor tympani tendon & Anterior ligament & Lateral ligament & Superior attachment \\
\hline \multicolumn{5}{|l|}{ In the malleus principal frame $(\mathrm{mm})$} \\
\hline Ear 1 & $(-0.1,-2.1,0.4)$ & $(-0.3,-0.9,0.0)$ & $(0.4,-0.6,-0.6)$ & $(0.5,2.3,0.5)$ \\
\hline Ear 2 & $(-0.3,-2.5,0.1)$ & $(-0.7,-1.5,0.0)$ & $(-0.6,1.0,-0.9)$ & $(0.6,2.0,0.5)$ \\
\hline Ear 3 & $(-0.4,-1.9,0.2)$ & $(-0.7,0.1,0.0)$ & $(0.5,-0.4,-0.8)$ & $(0.1,2.8,0.5)$ \\
\hline Ear 4 & $(-0.4,-2.2,-0.1)$ & $(-0.2,-0.6,-0.2)$ & $(0.8,-0.7,-0.8)$ & $(0.2,2.2,0.8)$ \\
\hline Mean & $(-0.3,-2.2,0.2)$ & $(-0.5,-0.7,-0.1)$ & $(0.3,-0.2,-0.8)$ & $(0.4,2.3,0.6)$ \\
\hline SEM & $(0.07,0.13,0.10)$ & $(0.13,0.33,0.05)$ & $(0.30,0.40,0.06)$ & $(0.12,0.17,0.08)$ \\
\hline Distance from the origin (mean \pm & $2.2 \pm 0.12$ & $1.0 \pm 0.23$ & $1.2 \pm 0.13$ & $2.4 \pm 0.15$ \\
\hline \multicolumn{5}{|l|}{ In the MIC principal frame $(\mathrm{mm})$} \\
\hline Ear 1 & $(-0.4,-2.5,-0.1)$ & $(-1.1,-1.5,-0.2)$ & $(-0.6,-0.9,-0.9)$ & $(-1.3,1.8,0.6)$ \\
\hline Ear 2 & $(-0.7,-2.9,-0.4)$ & $(-1.4,-2.0,-0.3)$ & $(-1.7,0.6,-0.9)$ & $(-0.9,1.6,0.7)$ \\
\hline Ear 3 & $(-0.1,-2.7,-0.1)$ & $(-1.5,-1.2,0.0)$ & $(-0.3,-0.9,-0.9)$ & $(-2.4,1.4,0.7)$ \\
\hline Ear 4 & $(-0.3,-2.7,-0.2)$ & $(-0.9,-1.2,-0.3)$ & $(-0.4,-0.9,-1.2)$ & $(-1.3,1.6,0.8)$ \\
\hline Mean & $(-0.4,-2.7,-0.2)$ & $(-1.2,-1.5,-0.2)$ & $(-0.8,-0.5,-1.0)$ & $(-1.5,1.6,0.7)$ \\
\hline SEM & $(0.13,0.08,0.07)$ & $(0.14,0.19,0.07)$ & $(0.32,0.38,0.07)$ & $(0.32,0.08,0.04)$ \\
\hline Distance from the origin (mean \pm SEM) & $2.7 \pm 0.10$ & $1.9 \pm 0.19$ & $1.6 \pm 0.16$ & $2.3 \pm 0.19$ \\
\hline
\end{tabular}


TABLE 4

Suspensory attachment orientations, in unit-directional vectors, of the malleus in the malleus principal frame (top) and in the MIC principal frame (bottom)

\begin{tabular}{|c|c|c|c|c|}
\hline Suspensory attachments & Tensor tympani tendon & Anterior ligament & Lateral ligament & Superior attachment \\
\hline \multicolumn{5}{|l|}{ In the malleus principal frame } \\
\hline Ear 1 & $(-0.68,0.32,0.66)$ & $(-0.86,-0.09,-0.50)$ & $(-0.58,-0.16,-0.80)$ & $(0.75,0.41,-0.52)$ \\
\hline Ear 2 & $(-0.39,0.14,0.91)$ & $(-0.99,0.14,-0.06)$ & $(-0.87,-0.20,-0.46)$ & $(0.27,0.89,-0.38)$ \\
\hline Ear 3 & $(-0.62,0.58,0.53)$ & $(-0.91,-0.35,-0.20)$ & $(0.92,-0.19,-0.35)$ & $(0.13,0.53,0.84)$ \\
\hline Ear 4 & $(-0.51,0.13,0.85)$ & $(-0.65,0.33,-0.69)$ & $(0.97,-0.18,-0.14)$ & $(0.32,0.95,0.07)$ \\
\hline Mean $^{\mathrm{a}}$ & $(-0.57,0.30,0.76)$ & $(-0.92,0.01,-0.39)$ & $(0.23,-0.38,-0.90)$ & $(0.47,0.88,0.00)$ \\
\hline Average angle deviation ${ }^{b}$ (degree) & 7.6 & 11.1 & 30.6 & 19.4 \\
\hline \multicolumn{5}{|l|}{ In the MIC principal frame } \\
\hline Ear 1 & $(-0.56,0.01,0.82)$ & $(-0.87,-0.36,-0.32)$ & $(-0.67,-0.30,-0.68)$ & $(0.42,0.68,-0.60)$ \\
\hline Ear 2 & $(-0.42,-0.08,0.90)$ & $(-0.99,-0.09,-0.08)$ & $(-0.79,-0.33,-0.52)$ & $(0.06,0.97,-0.24)$ \\
\hline Ear 3 & $(-0.78,0.06,0.62)$ & $(-0.55,-0.81,-0.21)$ & $(0.82,0.41,-0.41)$ & $(-0.12,0.43,0.90)$ \\
\hline Ear 4 & $(-0.12,-0.04,0.99)$ & $(-0.94,-0.03,-0.33)$ & $(0.81,0.25,-0.53)$ & $(-0.11,0.99,0.04)$ \\
\hline Mean $^{\mathrm{a}}$ & $(-0.49,-0.01,0.87)$ & $(-0.90,-0.35,-0.25)$ & $(0.08,0.01,-1.00)$ & $(0.08,1.00,0.03)$ \\
\hline Average angle deviation ${ }^{\mathrm{b}}$ (degree) & 8.5 & 11.0 & 28.8 & 20.2 \\
\hline
\end{tabular}

${ }^{a}$ Mean unit-directional vector is calculated from the sum of the unit-directional vectors of four samples.

${ }^{\mathrm{b}}$ Standard error of the mean of angles from the mean vector.

relatively large for the width of the contact surface on the lateral side (where the SEM is almost $20 \%$ of the mean value).

Table 6 shows the position vectors of the centroids of the two contact areas, with respect to the incus principal frame and with respect to the MIC principal frame. The centroid of the lateral contact $(2.8 \mathrm{~mm}$ from the origin of the incus principal frame and $3.8 \mathrm{~mm}$ from the origin of the MIC principal frame) is a little further away from the origin of the principal frames than the centroid of the medial contact ( $2.5 \mathrm{~mm}$ from the origin of the incus principal frame and $3.6 \mathrm{~mm}$ from the origin of the MIC principal frame).

Table 7 shows the orientations of the incus ligament as described by two unit-vectors normal to the contact planes (Fig. 11), with respect to the incus principal frame and with respect to the MIC principal frame. The four sample ears show slightly smaller variations in the incus ligament orientation with respect to the MIC principal frame than with respect to the incus principal frame. The vector normal to the medial contact plane shows larger variability with respect to both principal frames than the vector normal to the lateral contact plane. The vector normal to the lateral contact plane is nearly perpendicular to the $X Y$ plane of the MIC principal frame for ears 2 and $4\left(79^{\circ}\right.$ and $\left.82^{\circ}\right)$. The vector normal to the lateral contact plane deviates the most for ear 1 , while the vector normal to the medial contact plane deviates the most for ear 3 .

\section{Incudomalleal joint}

Figure 13 shows a thickness map of the IMJ for ear 1 . The maximum thickness appears on the medial side and is aligned along the anterior-posterior direction at this facet, which is also the case for the other ear samples.

The IMJ morphometry consisting of the average thicknesses, contact areas to the bones, and volume of the IMJ for the four ear samples are shown in Table 8.

The maximum thickness of the IMJ has a range from 0.27 to $0.39 \mathrm{~mm}$. Ear 3, which has distinctly heavier ossicles than the other samples (Table 1), has

TABLE 5

\begin{tabular}{|c|c|c|c|c|c|c|c|}
\hline \multicolumn{8}{|c|}{ Incus ligament dimensions } \\
\hline & & Ear 1 & Ear 2 & Ear 3 & Ear 4 & Mean & SEM \\
\hline \multicolumn{2}{|c|}{ Average thickness of the medial part (mm) } & 0.46 & 0.41 & 0.31 & 0.33 & 0.38 & 0.03 \\
\hline \multicolumn{2}{|c|}{ Average thickness of the lateral part $(\mathrm{mm})$} & 0.62 & 0.68 & 0.40 & 0.66 & 0.59 & 0.06 \\
\hline \multirow[t]{2}{*}{ Contact on the medial side of incus } & Width $\left(w_{M}\right)(\mathrm{mm})$ & 1.13 & 1.16 & 1.20 & 1.15 & 1.16 & 0.01 \\
\hline & Area $\left(\mathrm{mm}^{2}\right)$ & 0.44 & 0.51 & 0.35 & 0.74 & 0.51 & 0.08 \\
\hline \multirow{2}{*}{ Contact on the lateral side of incus } & Width $\left(w_{\mathrm{L}}\right)(\mathrm{mm})$ & 0.59 & 0.90 & 1.08 & 0.79 & 0.84 & 0.10 \\
\hline & Area $\left(\mathrm{mm}^{2}\right)$ & 0.25 & 0.68 & 0.62 & 0.75 & 0.57 & 0.11 \\
\hline
\end{tabular}




\begin{tabular}{|c|c|c|}
\hline \multicolumn{3}{|c|}{ TABLE 6} \\
\hline \multicolumn{3}{|c|}{ Attachment locations of the incus ligament: position vector } \\
\hline & Lateral contact & Medial contact \\
\hline \multicolumn{3}{|l|}{ In the incus principal frame $(\mathrm{mm})$} \\
\hline Ear 1 & $(2.9,0.6,-0.4)$ & $(2.5,0.4,0.4)$ \\
\hline Ear 2 & $(2.5,0.7,-0.3)$ & $(2.4,0.2,0.3)$ \\
\hline Ear 3 & $(2.6,1.1,0.0)$ & $(2.5,0.1,0.1)$ \\
\hline Ear 4 & $(2.0,1.8-0.3)$ & $(2.2,1.5,0.3)$ \\
\hline Mean & $(2.5,1.1,-0.3)$ & $(2.4,0.6,0.3)$ \\
\hline SEM & $(0.19,0.27,0.09)$ & $(0.07,0.32,0.06)$ \\
\hline Distance from the origin (mean \pm SEM) & $2.8 \pm 0.08$ & $2.5 \pm 0.05$ \\
\hline \multicolumn{3}{|l|}{ In the MIC principal frame $(\mathrm{mm})$} \\
\hline Ear 1 & $(3.7,0.9,-0.8)$ & $(3.5,0.8,-0.1)$ \\
\hline Ear 2 & $(3.3,1.1,-0.9)$ & $(3.3,0.8,-0.5)$ \\
\hline Ear 3 & $(3.2,2.3,-0.5)$ & $(3.2,1.4,-0.1)$ \\
\hline Ear 4 & $(3.2,1.9,-0.7)$ & $(3.4,1.7,-0.1)$ \\
\hline Mean & $(3.4,1.6,-0.7)$ & $(3.4,1.2,-0.2)$ \\
\hline SEM & $(0.12,0.33,0.09)$ & $(0.06,0.23,0.10)$ \\
\hline Distance from the origin (mean \pm SEM) & $3.8 \pm 0.08$ & $3.6 \pm 0.08$ \\
\hline
\end{tabular}

the largest maximum thickness $(0.39 \mathrm{~mm})$, and the ear 4 , which has the smallest ossicular mass among four samples, has the smallest maximum thickness $(0.27 \mathrm{~mm})$. The minimum thickness is close to zero and it appears between the lateral side and the center of the joint, and near the inferior side. The contact area on the incus side was larger than the contact area on the malleus side for all four ear samples, but the amount of the difference varies for each ear samples. Ear 4 shows little difference $\left(0.05 \mathrm{~mm}^{2}\right)$ between the two areas, but ears $1\left(0.49 \mathrm{~mm}^{2}\right), 2\left(0.53 \mathrm{~mm}^{2}\right)$, and 3 $\left(0.89 \mathrm{~mm}^{2}\right)$ show relatively large differences. The SEM is about $10 \%$ of the mean value for both areas. Variations in the joint volume are large, and the SEM is more than $20 \%$ of the mean value. Ear 3, which has the largest bone mass, maximum thickness, and largest contact areas, has the largest IMJ volume $\left(1.93 \mathrm{~mm}^{3}\right)$, which is more than twice as large as the volume of ear 1 , which has the smallest volume $\left(0.90 \mathrm{~mm}^{3}\right)$ among the four ear samples.

\section{DISCUSSION}

\section{Tensor tympani tendon}

The tensor tympani tendon has the most consistent dimensions, attachment position, and orientation of the soft-tissue structures examined in this study. In the work by Beer et al. (1996), where dimensions of the suspensory attachments were measured using a microscope, the diameter and the length of the tensor tympani tendon were found to be 1.0 and $3.9 \mathrm{~mm}$, whereas in the current study, we obtained $0.7-0.9 \mathrm{~mm}$ (diameter on the malleus surface - diameter on the

TABLE 7

\begin{tabular}{|c|c|c|}
\hline \multicolumn{3}{|c|}{ Orientation of the incus ligament: unit-directional vectors normal to two contact areas } \\
\hline & Normal to lateral contact $\left(\mathbf{n}_{L}\right)$ & Normal to medial contact $\left(\mathbf{n}_{M}\right.$ \\
\hline \multicolumn{3}{|l|}{ In the incus principal frame } \\
\hline Ear 1 & $(0.70,0.35,-0.62)$ & $(0.25,-0.45,0.86)$ \\
\hline Ear 2 & $(0.41,0.38,-0.83)$ & $(0.30,-0.49,0.82)$ \\
\hline Ear 3 & $(0.31,0.57,-0.76)$ & $(0.46,-0.86,0.20)$ \\
\hline Ear 4 & $(0.04,0.23,-0.97)$ & $(0.34,-0.26,0.90)$ \\
\hline Mean $^{\mathrm{a}}$ & $(0.38,0.40,-0.83)$ & $(0.36,-0.55,0.75)$ \\
\hline Angle deviation from the mean vector ${ }^{\mathrm{b}}$ (degrees) & 8.7 & 11.0 \\
\hline \multicolumn{3}{|l|}{ In the MIC principal frame } \\
\hline Ear 1 & $(0.60,0.15,-0.79)$ & $(0.38,-0.20,0.90)$ \\
\hline Ear 2 & $(0.18,0.07,-0.98)$ & $(0.52,-0.16,0.84)$ \\
\hline Ear 3 & $(0.14,0.35,-0.92)$ & $(0.64,-0.66,0.39)$ \\
\hline Ear 4 & $(0.06,-0.11,-0.99)$ & $(0.33,0.00,0.95)$ \\
\hline Mean $^{\mathrm{a}}$ & $(0.26,0.12,-0.96)$ & $(0.50,-0.27,0.83)$ \\
\hline Angle deviation from the mean vector ${ }^{b}$ (degrees) & 7.8 & 9.8 \\
\hline
\end{tabular}

${ }^{\mathrm{a}}$ Mean unit-directional vector is calculated from the sum of four unit-directional vectors.

${ }^{\mathrm{b}}$ Standard error of the mean of angles from the mean vector. 


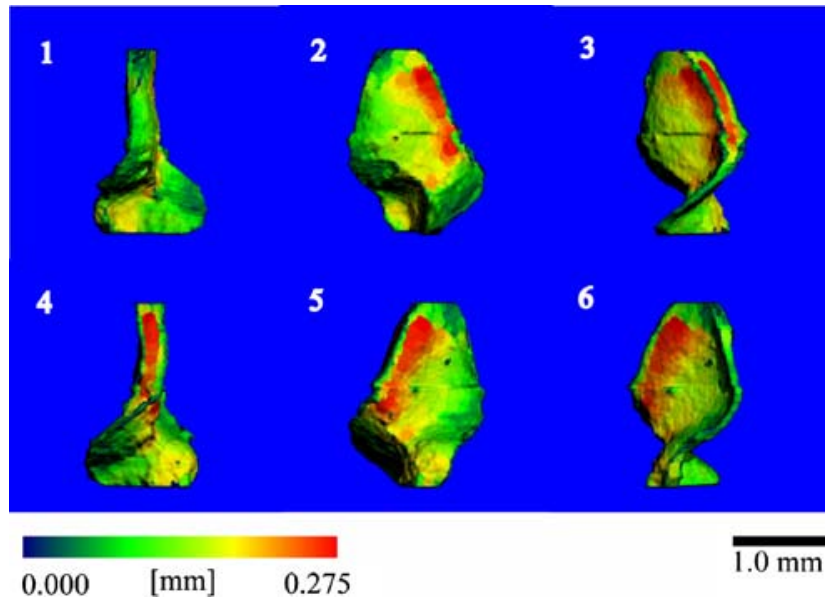

FIG. 13. Thickness map of the saddle-shaped incudomalleal joint (ear 1). Each subplot is a clockwise rotation of $60^{\circ}$ steps. The thickness is calculated by the distance transformation (DT) method, which fits spheres into the volume. The color map shows that the thickness varies from about $0.05 \mathrm{~mm}$ (dark green) to $0.275 \mathrm{~mm}$ (red). Superior is up while inferior is down.

middle-ear cavity bone) and $2.2 \mathrm{~mm}$ as the equivalent diameter and the length of the tensor tympani tendon. The large difference in the measured lengths may be because of differences in the portion of the tensor tympani tendon that was measured. We measured the length of the portion that lies outside the bony regions, and Beer et al. did not clearly describe which portion they used for their measurement. The attachment location of the tensor tympani tendon to the malleus is superior-inferior to the center of mass and is aligned on the $X Z$ plane of the MIC principal frame. Considering its position and orientation, it is expected that the tensor tympani tendon exerts forces that counter the hinge-like rotation of the MIC about the anterior-posterior direction, which is considered the primary motion of the MIC at low frequencies.

\section{Anterior ligament of the malleus}

The present dimensional measurements of the anterior ligament of the malleus $(0.8-0.11 \mathrm{~mm}$ in diameter and $2.1 \mathrm{~mm}$ in length) differ somewhat from the corresponding values of Beer et al. (0.7 $\mathrm{mm}$ in diameter and $2.8 \mathrm{~mm}$ in length). The attachment position of the anterior ligament exhibits less variation with respect to the MIC principal frame than with respect to the malleus principal frame, and is aligned approximately along the axis in the anterior-posterior direction with respect to both principal frames. The relatively large crosssection areas of the anterior ligament can be a source of torsion and bending stiffness about the axis in the anterior-posterior direction as well as longitudinal stiffness along the same direction. The fact that this ligament covers the anterior process of the malleus enhances its role as a source of torsion and bending stiffness.

\section{Lateral ligament of the malleus}

Dimensions of the lateral ligament are similar for all ear samples with the exception of ear 2. While dimensions of the lateral ligament were not reported by Beer et al., it is likely that the posterior ligament of the malleus in that study is the same as the lateral ligament in the present work, considering our observation that the lateral ligament extends to the posterior direction for some ear samples. Assuming that the present lateral ligament is equivalent to the posterior ligament in Beer et al., the lengths show relatively small differences $(2.0 \mathrm{~mm}$ in the present study and $2.8 \mathrm{~mm}$ in Beer et al.), while the corresponding equivalent diameters shows nearly a factor of two difference $(0.5-0.6 \mathrm{~mm}$ in the present study vs $1.0 \mathrm{~mm}$ in Beer et al.). As described in the "Results" section, this ligament has a rectangular-shaped crosssection for some ear samples. Therefore, the equivalent diameter for some ear samples may not be accurately measurable by light microscopic methods because the apparent width of the ligament varies with the viewing direction. The lateral ligament shows the largest variation in its attachment position and orientation (Fig. 12). The angle between the lateral ligaments of ears 2 and 4 is as large as $138^{\circ}$ within the malleus principal frame.

\section{Superior attachment of the malleus}

Ear 2 also shows the biggest difference in the length of the superior attachment of the malleus. Between the

TABLE 8

\begin{tabular}{|c|c|c|c|c|c|c|}
\hline \multicolumn{7}{|c|}{ Incudomalleal joint morphometry in terms of thickness, contact-to-bone area, and volume } \\
\hline & Ear 1 & Ear 2 & Ear 3 & Ear 4 & Mean & SEM \\
\hline Maximum thickness (mm) & 0.28 & 0.35 & 0.39 & 0.27 & 0.32 & 0.029 \\
\hline Minimum thickness (mm) & 0.05 & 0.05 & 0.05 & 0.03 & 0.04 & 0.005 \\
\hline Contact area onto the malleus $\left(\mathrm{mm}^{2}\right)$ & 4.95 & 5.28 & 7.42 & 5.70 & 5.84 & 0.55 \\
\hline Contact area onto the incus $\left(\mathrm{mm}^{2}\right)$ & 5.44 & 5.81 & 8.31 & 5.75 & 6.33 & 0.67 \\
\hline Volume $\left(\mathrm{mm}^{3}\right)$ & 0.90 & 1.31 & 1.93 & 1.13 & 1.24 & 0.28 \\
\hline
\end{tabular}


present study and Beer et al., the lengths of the superior attachment of the malleus are consistent (1.3 and $1.4 \mathrm{~mm}$, respectively), but the diameters show large differences between the two studies $(0.2-0.3 \mathrm{~mm}$ for the present study vs $0.7 \mathrm{~mm}$ for Beer et al.). The superior attachment is anchored to the superior part of the malleus and is aligned along the $Y$-axis (inferior-superior direction) of the MIC principal frame. Therefore, this attachment may provide stiffness for translational motions in the inferiorsuperior direction. However, its thin diameter suggests that its stiffness value is relatively small.

\section{Incus ligament}

The incus ligament exhibits large variations in dimensions and incus surface attachment position. Previous work (Wolff et al. 1957, from microscopic photos of horizontal cuts) has reported 0.29 and $0.5 \mathrm{~mm}$ as the thicknesses of the medial and lateral parts, respectively, with our values $(0.38$ and $0.59 \mathrm{~mm})$ only slightly larger. The work by Wolff et al. also reported 0.8 and $0.53 \mathrm{~mm}$ as the widths of the medial and lateral parts of the incus ligament, respectively, which is smaller than our values (1.16 and $0.84 \mathrm{~mm})$. However, the values by Wolff et al. were measured on a horizontally projected plane at a specific location such that the actual values for the width and maximum thickness should be larger than their reported values.

The incus ligament is oriented more consistently than the suspensory attachments of the malleus. It is known that the anterior ligament of the malleus and the incus ligament are attachments to the outer middleear cavity wall, oriented along the anterior-posterior rotational axis of the MIC (Fig. 12), which at low frequencies is the primary axis for the rotation of the MIC (Wever and Lawrence 1954; Békésy 1960). This rotational axis does not coincide with the principal axis of the MIC along the anterior-posterior direction, which passes through the center of mass of the MIC.

A notch in the short process of the incus was observed in three out of the four temporal bone samples (Fig. 4, right panel). The presence of this notch was previously reported (Arensburg and Nathan 1971; Unur et al. 2002), and may prove to be of some functional importance because it enhances the connection between the lateral and the medial parts of the incus ligament. However, its mechanical effects on incus vibration have not yet been investigated. An alternative nonmechanical function is that the notch is one of the access routes for incus vascularization.

\section{Incudomalleal joint}

Dimensions of the IMJ vary with ear samples and are approximately proportional to the ossicular mass
(Fig. 14). This suggests that the motion at the IMJ varies with ear samples because the ossicular mass varies across ear samples (Table 1 ).

Wolff et al. (1957) reported 0.02 and $0.36 \mathrm{~mm}$ as the minimum and maximum joint thicknesses, which are similar to the present values of 0.04 and $0.32 \mathrm{~mm}$. For all four ear samples, thicknesses on the lateral and medial sides are larger than on the superior and lateral sides (Fig. 13). Rotational motions about the axes along the anterior-posterior and the lateralmedial directions cause compression and elongation to the thinner part of the IMJ on the superior and inferior sides, while rotational motions about the axis along the inferior-superior direction cause compression and elongation to the thicker part of the IMJ on the medial and lateral sides. In the thinner part of the IMJ more force is required to compress and elongate a smaller depth of fluid, so the IMJ more easily allows rotation about the axis along the inferior-superior direction than rotations around the other two axes. Rotational motions about the axes along the anterior-posterior and superior-inferior directions reported by Willi et al. (2002) are consistent with expectations based on the anatomy of the IMJ in that the rotational motion about the axis along the superior-inferior direction is larger than the rotational motion about the axis oriented along the anterior-posterior direction below $1 \mathrm{kHz}$. In addition, the translational motion in the inferior-superior direction will be easier than
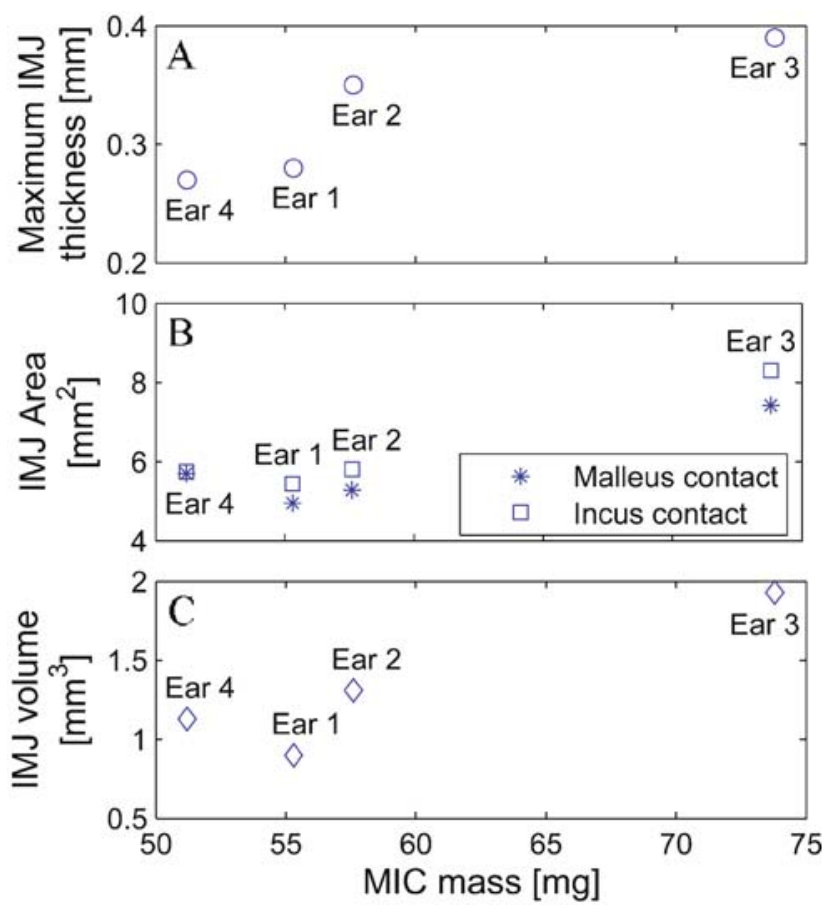

FIG. 14. Relationship between MIC mass and incudomalleal joint (IMJ) dimensions. These are $\mathbf{A}$ the maximum thickness, $\mathbf{B}$ area on the malleus and incus interfaces, and $\mathbf{C}$ the IMJ volume. 
other translational motions because the translational motion in this direction is mainly opposed by shear friction between the joint fluid and the bone surfaces and shear deformation of the fibrous structure on the joint boundary, while other translational motions are more opposed by normal pressure forces and elongation of the fibrous structure on the joint boundary.

\section{Isolating the MIC}

In developing an understanding and ultimately a biomechanical model of the human middle ear, portions of the middle ear were removed in a stepwise manner to decouple their functions from those of other surrounding components to better reveal their individual roles. This methodology has been used in the past by Wever and Lawrence (1954), Tonndorf and Pastaci (1986), Puria and Allen (1998), and others. The underlying assumption in such approaches is that the biomechanical properties of the reduced system are not altered from its operation in situ in the normal condition.

In the normal middles ear, it is assumed that preloads in suspensory structures are present and those preloads are balanced with eardrum and stapes preloads. The pulling forces of the eardrum along the medial-to-lateral direction will cause rotation and translation of the MIC about an axis along the anterior-posterior direction, resulting in pretension in the tensor tympani tendon and twisting in the anterior ligament and the incus ligament. Such pretensions will affect the positions of the bones, causing the suspensory attachments to have different orientations and size relative to the middle-ear bones. These effects have yet to be investigated and will likely alter the baseline morphometry presented in this study. In models of the normal ear, the effects of preload in suspensory attachments on middle-ear mechanics can and should be taken into account.

\section{CONCLUSION}

Dimensions and positions of the soft tissue structures in the human MIC were examined and found to vary significantly across ear samples. These large variations prevent a single middle-ear model from being applicable to all ears, so biomechanical models of the MIC should be based on the specific anatomical features of individual ears. Micro-CT imaging is a modality that provides nondestructive and relatively fast morphometry of individual ear samples and allows calculation of principle frames used for reference.

Through observations of the positions and orientations of the suspensory attachments to the MIC
(Fig. 12), it is expected that the primary rotation of the MIC about the axis along the anterior-posterior direction, the classical hinging motion, is mainly because of the tensor tympani tendon, the anterior ligament of the malleus, and the incus ligament. Those suspensory attachments are presumed to be predeformed for force balance with preload by the eardrum. The superior attachment of the malleus will provide a source of stiffness to translational motion of the MIC along the inferior-superior direction, together with the anterior ligament of the malleus and the incus ligament. Other translational and rotational motions of the MIC are also constrained by combinations of the suspensory attachments. The mechanics of these attachments are likely to be most important at low and middle frequencies. At high frequencies, rotational inertia is lowest through the inferior-superior axis of the malleus in animals such as human and cat, where the incus-malleus joint is mobile. The rotational motion of the malleus is transferred through the saddle-shaped flexible synovial joint to the incus through an angle change forming a biological gear (Puria et al. 2007b).

\section{ACKNOWLEDGEMENT}

Work supported in part by a grant from NIDCD of NIH (DC005960). We thank Dr. Toshiki Maetani and Dr. Yoshitaka Shimizu for their help with temporal bone dissections, Minyong Shin and Kevin O'Connor for valuable comments and suggestions, and Dr. Seungbum Koo and for assistance with micro-CT imaging and analyses.

\section{REFERENCES}

Arensburg B, Nathan H. Observations on a notch in the short (superior or posterior) process of the incus. Acta. Anat. (Basel), 78(1):84-90, 1971.

Beer HJ, Borniz M, Drescher J, Schmidt R, Hardtke HJ, Hofmann G, Vogel U, Zahnert Th, Hüttenbrink KB. Finite element modeling of the human eardrum and application. Proceeding of the International Workshop on MEMRO: 40-47, 1996.

Beer HJ, Borniz M, Drescher J, Hardtke HJ, Schmidt R, Hofmann G, Vogel U, Zahnert T, Hüttenbrink KB. Modeling of components of the human middle ear and simulation of their dynamic behavior. Audiol. Neurootol. 4:156-162, 1999.

Békésy G. Series in Psychology: Experiment in Hearing. McGrawHill Book Company, pp. 95-126, 1960.

Brooks R, Di Chiro G. Beam hardening in x-ray reconstructive tomography. Phys. Med. Biol. 21(3):390-398, 1976.

Colbert EH, Morales M. Evolution of the Vertebrates: A History of the Backboned Animals through Time. 4th Edition. New York, Wiley, 1991.

Decraemer WF, Dirckx JJJ, Funnel WRJ. Three-dimensional modeling of the middle-ear ossicular chain using a commercial highresolution X-ray CT scanner. JARO 04:250-263, 2003. 
Donaldson JA, Duckert G, Lambert PM, Rubel Ee. Surgical Anatomy of the Temporal Bone. Philadelphia, Saunders, 1992.

Gan RZ, Sun Q, Dyer RK JR, Chang KH, Domer KJ. Threedimensional modeling of middle ear biomechanics and its application. Otol. Neurol. 23:271-280, 2002.

Gray H. Anatomy of the Human Body. Philadelphia, Lea \& Febiger, X.1d.3., 1918

Helmholtz H. On the Sensations of Tone. Mineola, NY, Dover Publications, 1968.

Hildebrand T, RüEgsegger P. A new method for the model independent assessment of thickness in three-dimensional images. J. Microsc. 185:67-75, 1997.

Kirikae J. The Middle Ear. Tokyo, University of Tokyo Press, 1960.

KoIKe T, WADA H, KobaYashi T. Modeling of the human middle ear using the finite element method. J. Acoust. Soc. Am. 111(3):1306-1317, 2002.

Lane JI, Witte RJ, Driscoll CLW, Camp JJ, Robb RA. Imaging microscopy of the middle and inner ear, Part I: CT microscopy. Clin. Anat. 17:607-612, 2004.

Marquet J. The incudo-malleal joint. J. Laryngol. Otol. 95:543$565,1981$.

Masterton B, Heffner H, Ravizza R. The evolution of human hearing. J. Acoust. Soc. Am. 45(4):966-985, 1969.

Puria S, Allen JB. Measurements and model of the cat middle ear: evidence of tympanic membrane acoustic delay. J. Acoust. Soc. Am. 104(6):3463-81, 1998.

Puria S, Sim JH, Shin M, Tuck-Lee J, Steele CR. Middle ear morphometry from cadaveric temporal bone micro-CT imaging. In: Eiber A and Huber A (eds) The 4th International Symposium on Middle Ear Mechanics in Research and
Otology, Zurich, Switzerland. Singapore, World Scientific Press, 2007a.

Puria S, Sim JH, Shin M, Steele CR. A gear in the middle ear. ARO Denver CO, 2007b.

Schuknecht HF. The middle ear. In: Pathology of the Ear. MA Harvard University Press, pp. 23-36, 1974.

Sim JH, Puria S, Steele CR. Imaging, physiology, and biomechanics of the malleus-incus complex. The 4th international symposium on middle ear mechanics in research and otology, Zurich, Switzerland, 2006.

Sim JH, Puria S, Steele CR. Calculation of inertia properties of the malleus-incus complex using micro-CT imaging. J. Mech. Mater. Struct. 2(8):1515-1524, 2007.

Tonndorf J, PAstaci H. Middle ear sound transmission: a field of early interest to Merle Lawrence. Am. J. Otolaryngol. 7(2):120-129, 1986.

UNUR E, ÜLgER H, EKÍNCI N. Morphometrical and morphological vibrations of middle ear ossicles in the newborn. Erciyes. Med. J. 24(2):57-63, 2002.

Wang G, Vannier MW. Computerized tomography. In: Webster JG (ed) Encyclopedia of Electrical and Electronics Engineering. New York, John Wiley \& Sons, 1998.

Weistenhöfer C, Hudde H. Determination of the shape and inertia properties of the human auditory ossicles. Audiol. Neurootol. 4:192-196, 1999.

Wever EG, Lawrence M. Physiological Acoustics. Princeton, Princeton University Press, 1954.

Willi UB, Ferrazzini MA, Huber AM. The incudo-malleolar joint and sound transmission losses. Hear. Res. 174:32-44, 2002.

Wolff D, Bellucci RJ, Eggston AA. Microscopic Anatomy of the Temporal Bone. Baltimore, Williams \& Wilkins, pp. 16-19, 1957. 